\title{
Charm at KEDR
}

V. V. Anashin ${ }^{a}$, O. V. Anchugov ${ }^{a}$, A. V. Andrianov ${ }^{a}$, K. V. Astrelina ${ }^{a}$, V. M. Aulchenko ${ }^{a, b}$, E. M. Baldin ${ }^{a, b}$, G. N. Baranov ${ }^{a, c}$, A. K. Barladyan ${ }^{a}$,

A. Yu. Barnyakov ${ }^{a, b, c}$, M. Yu. Barnyakov ${ }^{a, b, c}$, S. E. Baru ${ }^{a, b}$, I. Yu. Basok ${ }^{a}$,

E. A. Bekhtenev ${ }^{a}$, O. V. Belikov ${ }^{a}$, D. E. Berkaev ${ }^{a}$, A. E. Blinov ${ }^{a, b}$, V. E. Blinov ${ }^{a, b, c}$,

M. F. Blinov ${ }^{a}$, A. V. Bobrov ${ }^{a, b}$, V. S. Bobrovnikov ${ }^{a, b}$, A. V. Bogomyagkov ${ }^{a, b}$,

D. Yu. Bolkhovityanov ${ }^{a}$, A. E. Bondar ${ }^{a, b}$, A. R. Buzykaev ${ }^{a, b}$, P. B. Cheblakov ${ }^{a, b}$,

V. L. Dorohov ${ }^{a, c}$, S. I. Eidelman ${ }^{a, b, d}$, F. A. Emanov ${ }^{a, b}$, V. V. Gambaryan ${ }^{a}$,

S. A. Glukhov ${ }^{a, b}$, D. N. Grigoriev ${ }^{a, b, c}$, V. V. Kaminskiy, ${ }^{a, b}$, S. E. Karnaev ${ }^{a}$,

S. V. Karpov ${ }^{a}$, G. V. Karpov ${ }^{a}$, K. Yu. Karukina ${ }^{a, c}$, D. P. Kashtankin ${ }^{a}$,

P. V. Kasyanenko ${ }^{a, c}$, A. A. Katcin ${ }^{a, b}$, T. A. Kharlamova ${ }^{a, b, *}$, V. A. Kiselev ${ }^{a}$,

S. A. Kononov ${ }^{a, b}$, K. Yu. Kotov ${ }^{a}$, A. A. Krasnov ${ }^{a}$, E. A. Kravchenko ${ }^{a, b}$,

V. N. Kudryavtsev ${ }^{a, b}$, V. F. Kulikov ${ }^{a, b}$, G. Ya. Kurkin ${ }^{a, c}$, I. A. Kuyanov ${ }^{a}$,

E. B. Levichev ${ }^{a, c}$, P. V. Logachev ${ }^{a}$, D. A. Maksimov ${ }^{a, b}$, Yu. I. Maltseva ${ }^{a}$,

V. M. Malyshev ${ }^{a}$, A. L. Maslennikov ${ }^{a, b}$, O. I. Meshkov ${ }^{a, b}$, S. I. Mishnev ${ }^{a}$,

I. I. Morozov ${ }^{a, b}$, I. A. Morozov ${ }^{a}$, N. Yu. Muchnoi ${ }^{a, b}$, D. A. Nikiforov ${ }^{a}$, S. A. Nikitin ${ }^{a}$,

I. B. Nikolaev ${ }^{a, b}$, I. N. Okunev ${ }^{a}$, A. P. Onuchin ${ }^{a, b}$, S. B. Oreshkin ${ }^{a}$, V. V. Oreshonok ${ }^{a}$,

A. A. Osipov ${ }^{a, b}$, I. V. Ovtin ${ }^{a, b}$, A. V. Pavlenko ${ }^{a}$, S. V. Peleganchuk ${ }^{a, b}$, V. V. Petrov ${ }^{a}$,

P. A. Piminov ${ }^{a}$, S. G. Pivovarov ${ }^{a, c}$, N. A. Podgornov ${ }^{a}$, V. G. Prisekin ${ }^{a, b}$,

O. L. Rezanova ${ }^{a, b}$, A. A. Ruban ${ }^{a, b}$, G. A. Savinov ${ }^{a}$, A. G. Shamov ${ }^{a, b}$, L. I. Shekhtman ${ }^{a}$,

D. A. Shvedov ${ }^{a}$, B. A. Shwartz ${ }^{a, b}$, E. A. Simonov ${ }^{a}$, S. V. Sinyatkin ${ }^{a}$, A. N. Skrinsky ${ }^{a}$,

A. V. Sokolov ${ }^{a, b}$, E. V. Starostina ${ }^{a, b}$, D. P. Sukhanov ${ }^{a}$, A. M. Sukharev ${ }^{a, b}$,

A. A. Talyshev ${ }^{a, b}$, V. A. Tayursky ${ }^{a, b}$, V. I. Telnov ${ }^{a, b}$, Yu. A. Tikhonov ${ }^{a, b}$,

K. Yu. Todyshev ${ }^{a, b}$, A. G. Tribendis ${ }^{a, c}$, G. M. Tumaikin ${ }^{a}$, Yu. V. Usovi ${ }^{a}$, A. I. Vorobiov ${ }^{a}$,

V. N. Zhilich ${ }^{a, b}$, A. A. Zhukov ${ }^{a}$, V. V. Zhulanov ${ }^{a, b}$, A. N. Zhuravlev ${ }^{a, b}$

${ }^{a}$ Budker Institute of Nuclear Physics

11, akademika Lavrentieva prospect, Novosibirsk, 630090, Russia

${ }^{b}$ Novosibirsk State University

2, Pirogova street, Novosibirsk, 630090, Russia

${ }^{c}$ Novosibirsk State Technical University

20, Karl Marx prospect, Novosibirsk, 630092, Russia

${ }^{d}$ Lebedev Institute RAS

Leninsky pr. 53, 119991, Moscow, Russia

E-mail: t.a.kharlamova@inp.nsk.su.edu 
We review the recent results obtained by the KEDR experiment in the charmonium energy range. They include the measurements of $\mathrm{J} / \psi$ meson total and partial widths and exclusive branching fractions, study of $\mathrm{D}^{+}$and $\mathrm{D}^{0}$ meson masses and $\mathrm{R}$ between 1.8 and $7.0 \mathrm{GeV}$.

10th International Workshop on Charm Physics (CHARM2020)

31 May - 4 June, 2021

Mexico City, Mexico - Online 


\section{Introduction}

We report recent results from the KEDR detector at the VEPP-4M $\mathrm{e}^{+} \mathrm{e}^{-}$collider in the Budker Institute in Novosibirsk. VEPP-4M collider was designed to operate in the beam energy from 1 to $5 \mathrm{GeV}$ [1]. One of the main features of the VEPP-4M collider is possibility of precise energy determination with two methods: resonant depolarization method [2] and infrared light Compton backscattering method [3]. The KEDR detector is described in [4].

\section{Measurements of $\mathrm{J} / \psi$ meson total and partial widths}

Analysis on $\mathrm{J} / \psi$ meson widths measurement was based on the dataset with integrated luminosity of $230 \mathrm{nb}^{-1}$ at 11 energy points. That allowed to fit the resonant shape and determine non-resonant background contributions. To measure the width hadronic and electronic channels were considered simultaneously. We performed combined fit of the data and determined $\Gamma_{\mathrm{ee}} \cdot \mathrm{B}_{\mathrm{ee}}(\mathrm{J} / \psi), \Gamma_{\mathrm{ee}} \cdot \mathrm{B}_{\text {hadrons }}(\mathrm{J} / \psi)$ and $\Gamma_{\mathrm{ee}}(\mathrm{J} / \psi)[5]$.

The electronic width obtained in our analysis $\Gamma_{\mathrm{ee}}=5.550 \pm 0.056 \pm 0.089 \mathrm{keV}$ agrees well with the world average [6]. Figure 1 shows its comparison with the previous measurements [7-11] and lattice QCD calculations [12,13]. The values of $\Gamma_{\mathrm{ee}}(\mathrm{J} / \psi)$ in BESIII [7], CLEO [8] and BaBar [9] experiments were calculated from $\Gamma_{\text {ee }} \cdot B_{\mu \mu}(J / \psi)$ measured in the radiation process $\mathrm{e}^{+} \mathrm{e}^{-} \rightarrow \mu^{+} \mu^{-} \gamma$ with the $\mathrm{J} / \psi$ meson decaying to muon pair. Our measurement of leptonic width is direct measurement as the result from BES [11]. In the analysis we considered the inclusive hadronic channel as well as leptonic processes to extract partial widths of the $\mathrm{J} / \psi$ meson.

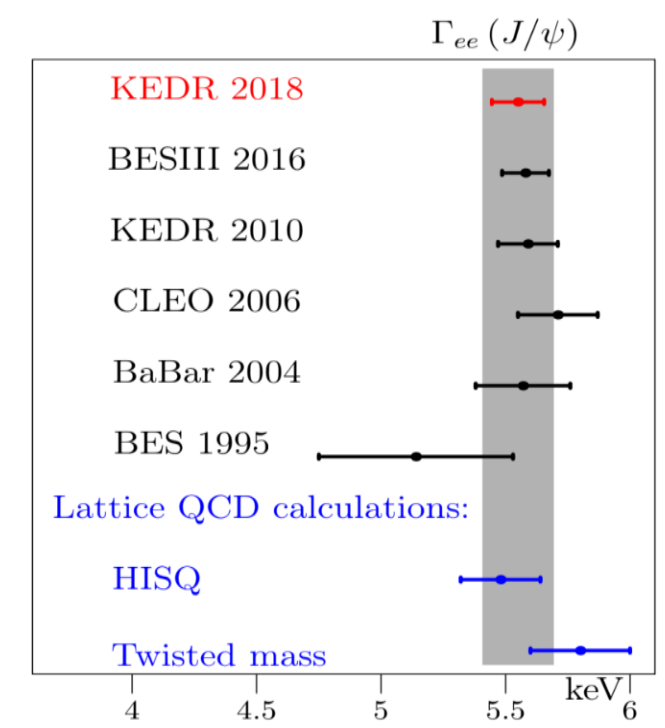

Figure 1: Comparison of the KEDR results with the previous measurements

Our result for the $\Gamma_{\text {ee }} \cdot B_{\text {hadrons }}(\mathrm{J} / \psi)$ value (Fig. 2) is consistent with and four times more precise than the previous direct measurement in the hadronic channel [11]. Figure 2 shows $\Gamma_{\mathrm{ee}} \cdot \mathrm{B}_{\text {hadrons }}(\mathrm{J} / \psi)$ and $\Gamma_{\mathrm{ee}} \cdot \mathrm{B}_{\mathrm{ee}}(\mathrm{J} / \psi)$ comparison with the previous measurements [11, 14-16]. 

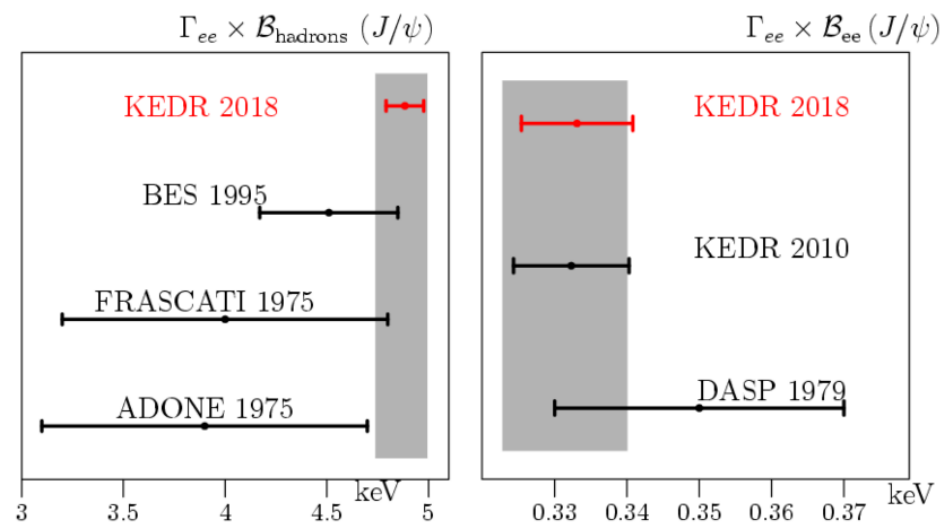

Figure 2: Measurements of $\Gamma_{\mathrm{ee}} \cdot \mathrm{B}_{\text {hadrons }}(\mathrm{J} / \psi)$ and $\Gamma_{\mathrm{ee}} \cdot \mathrm{B}_{\mathrm{ee}}(\mathrm{J} / \psi)$

As a continuation of this analysis the total and hadronic widths were also measured [5]. The total width obtained is a direct measurement involving data only from KEDR experiment. New result on $\Gamma_{\text {hadr }}(\mathrm{J} / \psi)$ is consistent with and 4 times more precise that the previous direct measurement of the hadronic width by BES collaboration [11]. In Figure 3 we present measured $\Gamma(\mathrm{J} / \psi)$ and $\Gamma_{\text {hadrons }}(\mathrm{J} / \psi)$ values with those obtained in previous experiments.
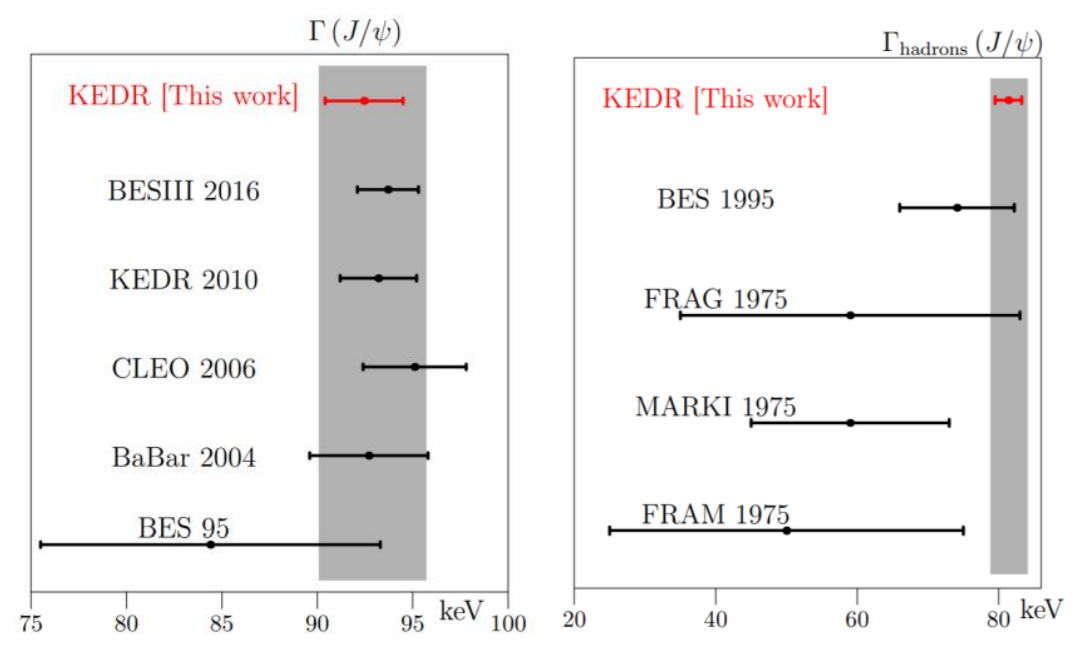

Figure 3: Measurements of $\Gamma(\mathrm{J} / \psi)$ and $\Gamma_{\text {hadrons }}(\mathrm{J} / \psi)$

\section{Decays $\mathbf{J} / \psi \rightarrow 2\left(\pi^{+} \pi^{-}\right) \pi^{0}, \mathrm{~K}^{+} \mathrm{K}^{-} \pi^{+} \pi^{-} \pi^{0}, 2\left(\pi^{+} \pi^{-}\right), \mathrm{K}^{+} \mathrm{K}^{-} \pi^{+} \pi^{-}$}

KEDR collaboration is also measuring branching fractions of $\mathrm{J} / \psi \rightarrow 2\left(\pi^{+} \pi^{-}\right) \pi^{0}, \mathrm{~K}^{+} \mathrm{K}^{-} \pi^{+} \pi^{-}$ $\pi^{0}, 2\left(\pi^{+} \pi^{-}\right), \mathrm{K}^{+} \mathrm{K}^{-} \pi^{+} \pi^{-}$. Our preliminary results on inclusive decay modes compared with previous experiments are shown on Figure 4. The analysis uses the statistics of about $1.3 \mathrm{pb}^{-1}$ at the peak of the $\mathrm{J} / \psi$ resonance that corresponds to 5.2 million of produced $\mathrm{J} / \psi$ mesons and $82.3 \mathrm{nb}^{-1}$ for background estimation. 

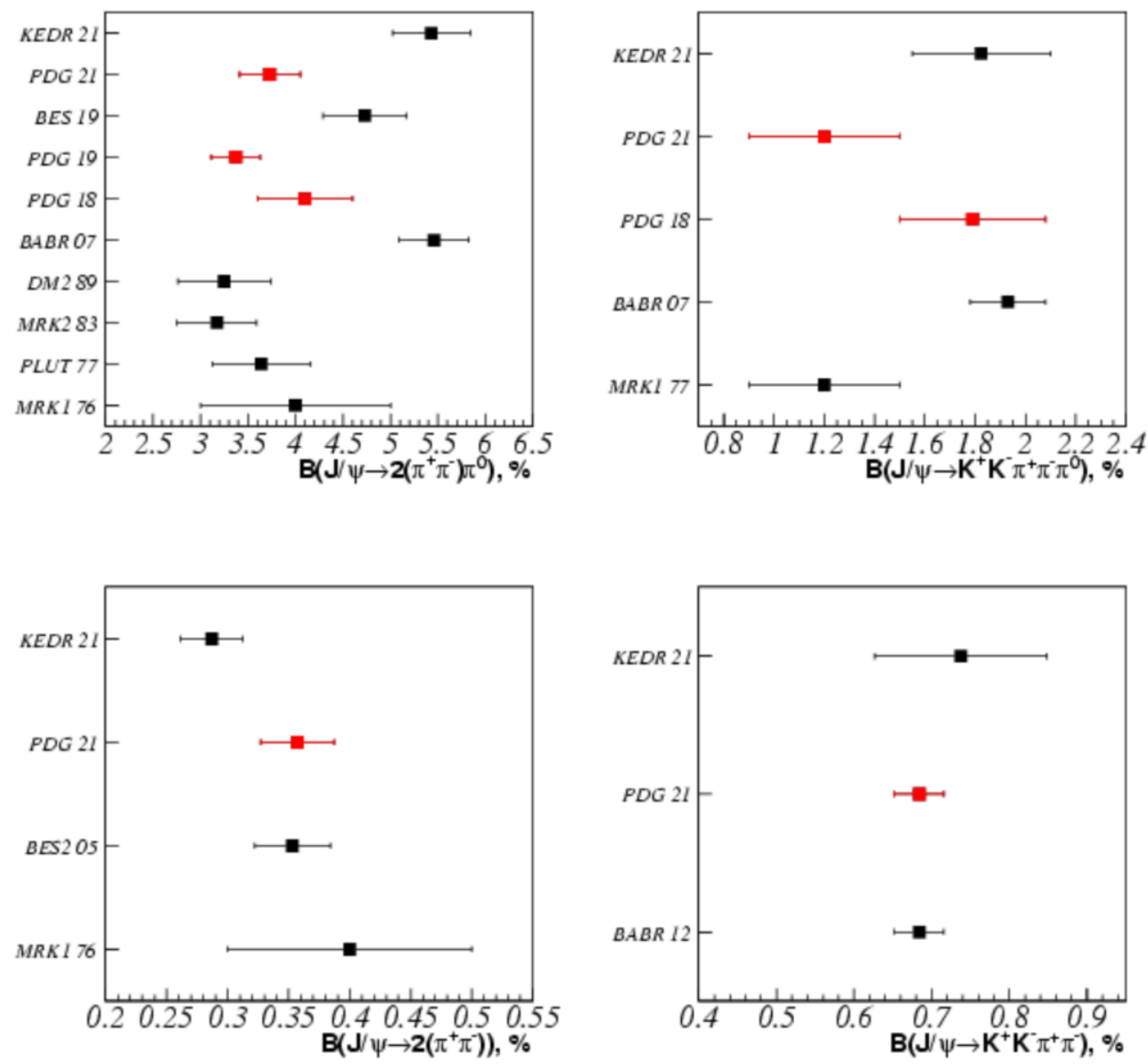

Figure 4: Summary of preliminary results on branching fractions of $J / \psi$ meson inclusive decay modes

\section{4. $D$ meson masses}

Neutral and charged D mesons are the ground states in the family of open charm mesons. Measurement of their masses provides a mass scale for the heavier excited states. D-meson mass is important for DD* threshold determination. This knowledge affects understanding of the $\chi_{\mathrm{cl}}(3872)(\mathrm{X}(3872))$ nature [17].

Measurement of $\mathrm{D}$ meson masses is performed using the near-threshold $\mathrm{e}^{+} \mathrm{e}^{-} \rightarrow \mathrm{D} \overline{\mathrm{D}}$ production with full reconstruction of one of the D mesons. Neutral D mesons are reconstructed in the $\mathrm{K}^{-} \pi^{+}$final state, charged $\mathrm{D}$ mesons are reconstructed in the $\mathrm{K}^{-} \pi^{+} \pi^{+}$final state. For selections beam - constrained mass $M_{b c}=\sqrt{E_{\text {beam }}^{2}-\left(\sum_{i} \vec{p}_{i}\right)^{2}}$ and center-of-mass (CM) energy difference $\Delta E=\sum_{i} \sqrt{\left(m_{i}^{2}+p_{i}^{2}\right)}-E_{\text {beam }}$ are calculated in every event, where $E_{\text {beam }}$ is the energy of colliding beams in the CM frame, $m_{i}$ and $\vec{p}_{i}$ are the masses and momenta of the D meson decay products. Additional kinematic parameter $\Delta|p|$ determined as the difference of the absolute values of momenta for $\mathrm{D}$ decay products in the $\mathrm{CM}$ frame is used for $\mathrm{D}^{0}$ selection. For the signal events the parameter $\Delta E$ is close to zero. In our analysis, we select a relatively wide region of $\Delta E$ and $M_{b c}$ close to $M_{D}$, then perform a fit of the event density with D mass as one of the parameters, 
with the background contribution taken into account. Results of selection of the $\mathrm{D}^{0}$ and $\mathrm{D}^{ \pm}$meson candidates are shown in Figures 5 and 6 correspondingly.
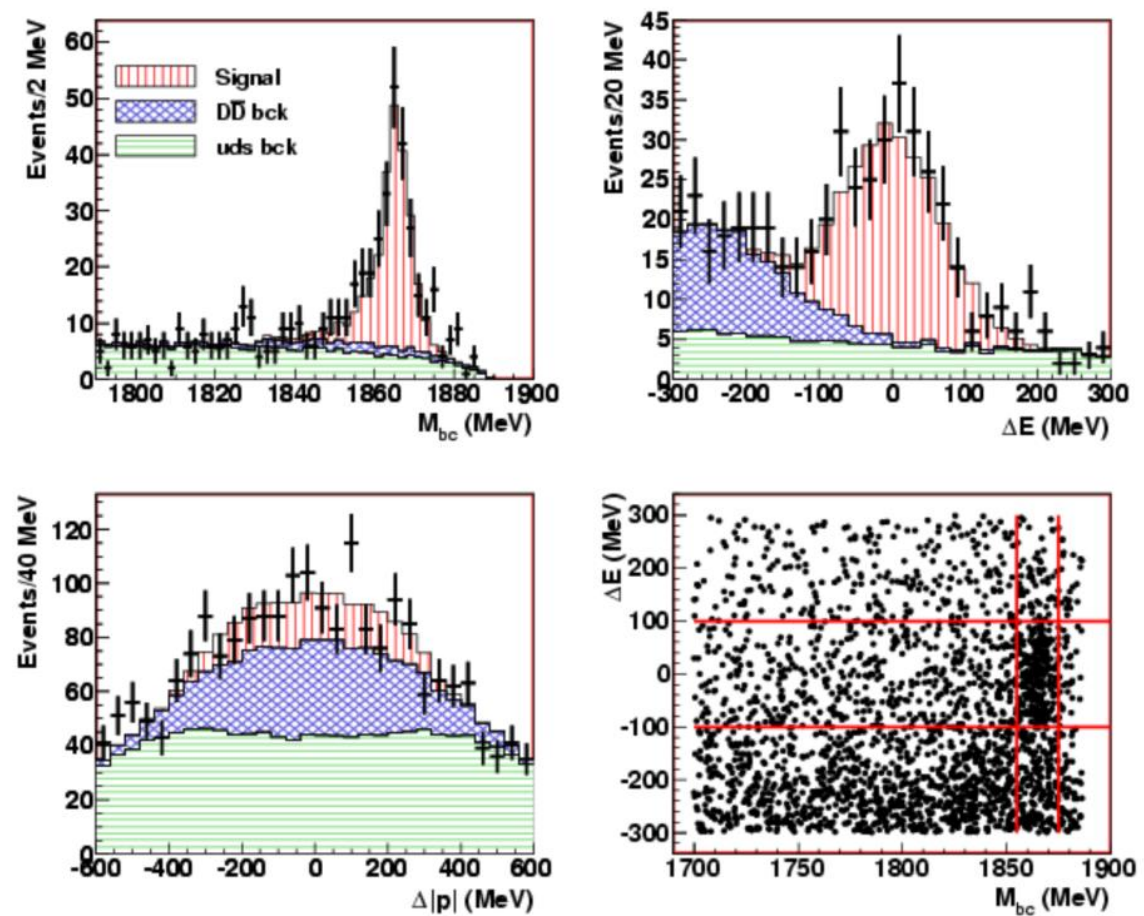

Figure 5: Selection of $\mathrm{D}^{0}$ candidates
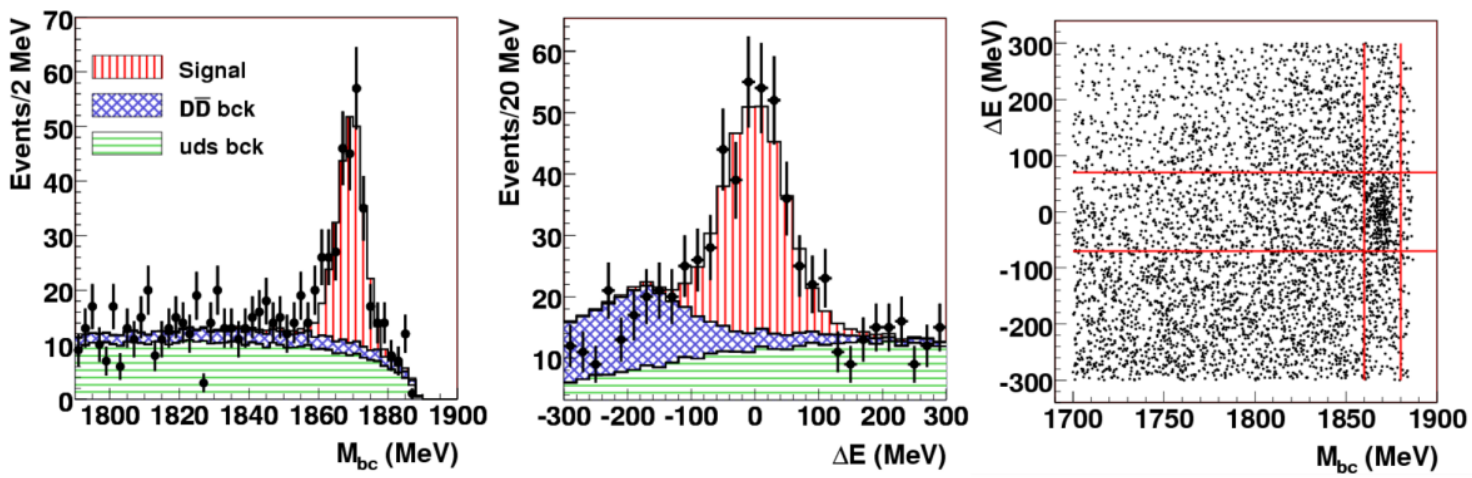

Figure 6: Selection of $\mathrm{D}^{ \pm}$candidates

D-meson masses were measured at KEDR at 2010 [18]. But now new analysis is ongoing with increased statistics, and we aim to increase accuracy about $20 \%$ compared to previous measurement from KEDR experiment.

\section{4. $R$ measurements between 1.8 and $7.0 \mathrm{GeV}$}

$\mathrm{R}$ is the ratio of the radiatively corrected total cross section of electron-positron annihilation into hadrons and muon pairs. $\mathrm{R}$ is crucial in various precision tests of Standard Model determination of the running strong coupling constant $\alpha_{s}(s)$ and heavy quark masses, evaluation 
of the hadronic contribution to the anomalous magnetic moment of the muon $(\mathrm{g}-2)_{\mu}$ and calculation of the value of the electromagnetic fine structure constant at the $Z^{0}$ peak $\alpha\left(M_{Z}{ }^{2}\right)$ [19].

On Figure 6 the most recent measurement of $R$ are presented in the energy range between 1.8 and $3.8 \mathrm{GeV}$. $\mathrm{R}$ was measured at KEDR in this energy range at 13 equidistant points between $1.84-3.05 \mathrm{GeV}[20]$. The achieved accuracy is about or better that $3.9 \%$ at the most of energy points. For the energies above $\mathrm{J} / \psi$ resonance there were 9 equidistant points with total error of about or better that $2.6 \%$ [21].

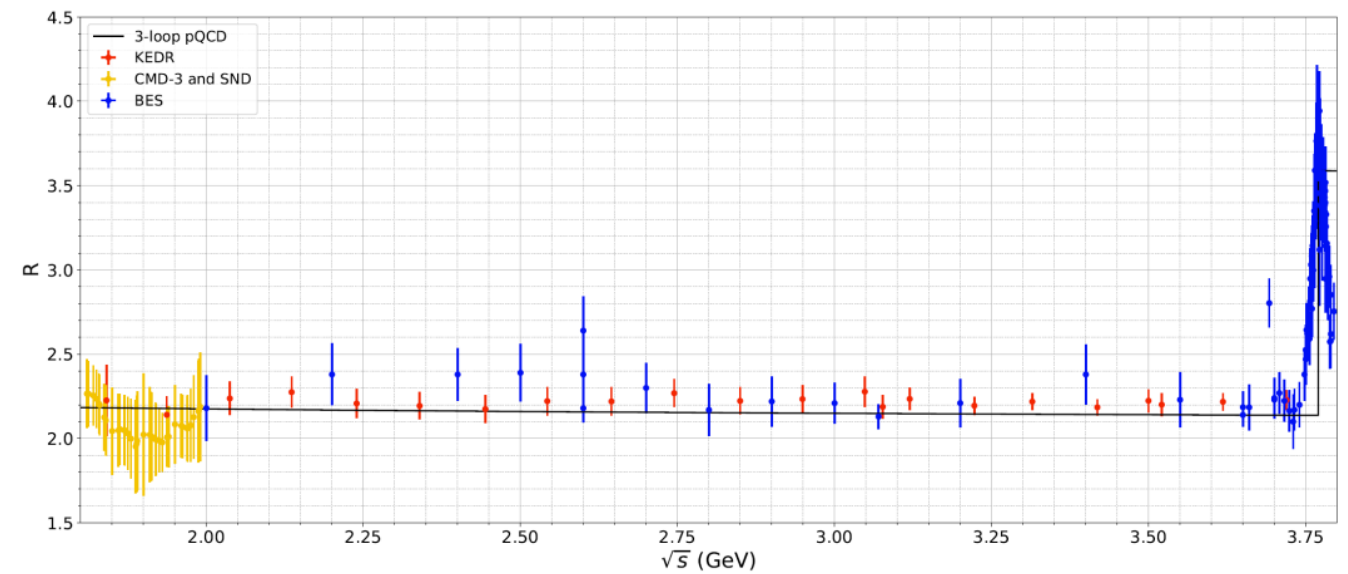

Figure 6: R measurement between 1.8 and $3.8 \mathrm{GeV}$

New data taking was done in the energy range from 4.7 to $7 \mathrm{GeV}$ with integrated luminosity $13.7 \mathrm{pb}^{-1}$. The range is interesting because there are no published data between $5 \mathrm{GeV}$ and $6.96 \mathrm{GeV}[22,23]$. VEPP-4M collected statistics at 17 equidistant points in this energy range. Expected total uncertainty is expected to be about $3 \%$ with systematical uncertainty of about $2.5 \%$.

\section{Summary}

New precise measurement of $\mathrm{J} / \psi$ total and leptonic width is presented. KEDR measured the $\mathrm{R}$ values at 22 center-of-mass energies between 1.84 and $3.72 \mathrm{GeV}$. Analysis of data in the energy range between 4.56 and $6.96 \mathrm{GeV}$ was started, expected accuracy is less than $3 \%$. New analyzes of the D-meson masses and branching fractions of $\mathrm{J} / \psi \rightarrow 2\left(\pi^{+} \pi^{-}\right) \pi^{0}, \mathrm{~K}^{+} \mathrm{K}^{-} \pi^{+} \pi^{-} \pi^{0}, 2\left(\pi^{+} \pi^{-}\right), \mathrm{K}^{+} \mathrm{K}^{-} \pi^{+} \pi^{-}$ are ongoing.

\section{References}

[1] V. V. Anashin et al., VEPP-4M Collider: Status and Plans, Proc. of EPAC 98*, Stockholm (1998) 400.

[2] A. D. Bukin et al., Absolute calibration of beam energy in the storage ring, $\Phi$-meson mass measurement, Preprint IYF-75-64, 1975B.

[3] G.Ya. Kezerashvili et al., A Compton source of high-energy polarized tagged gamma-ray beams. The ROKK-1M facility, Nucl. Instrum. Meth. B145 (1998) 40.

[4] V. V. Anashin et al., KEDR collaboration, The KEDR detector, Phys. of Part. and Nucl. 44 (2013) 657. 
[5] V. V. Anashin et al., KEDR collaboration, Measurement of $\Gamma_{e e}(J / \psi)$ with KEDR detector, JHEP 05 (2018) 119, arXiv:1801.01958. V. V. Anashin et al., KEDR collaboration, Addendum to: Measurement of $\Gamma_{e e}(J / \psi)$ with KEDR detector, JHEP 07 (2020) 112.

[6] P.A. Zyla et al. (Particle Data Group), Review of Particle Physics, Prog. Theor. Exp. Phys. 2020, 083C01 (2020) and 2021 update.

[7] M. Ablikim et al., BES Collaboration, Measurement of the leptonic decay width of J/ $\psi$ using initial state radiation, Phys. Lett. B. 761 (2016) 98.

[8] G. S. Adams et al., CLEO Collaboration, Measurement of $\Gamma_{e e}(J / \psi), \Gamma_{t o t}(J / \psi)$, and $\Gamma_{e e}\left(\psi(2 S) / \Gamma_{e e}(J / \psi)\right.$, Phys. Rev. D. 73 (2006) 051103.

[9] B. Aubert et al., BaBar Collaboration, J/ $\psi$ production via initial state radiation in $e^{+} e^{-} \rightarrow \mu^{+} \mu^{-} \gamma$ at an $e^{+} e^{-}$center-of-mass energy near 10.6 GeV, Phys. Rev. D. 69 (2004) 011103.

[10] V. V. Anashin et al., KEDR Collaboration, Measurement of $\Gamma_{e e}(J / \psi) \cdot B r\left(J / \psi \rightarrow e^{+} e^{-}\right)$and $\Gamma_{e e}(J / \psi) \cdot \operatorname{Br}\left(J / \psi \rightarrow \mu^{+} \mu^{-}\right)$, Phys. Lett. B. 685 (2010) 134.

[11] J. Z Bai et al., BES Collaboration, A measurement of J/ $\psi$ decay widths, Phys. Lett. B. 355 (1995) 374.

[12] G. C. Donald et al., HPQCD Collaboration, Precision tests of the J/ $\psi$ from full lattice QCD: Mass, leptonic width, and radiative decay rate to $\eta_{c}$, Phys. Rev. D. 86 (2012) 094501.

[13] D. Becirevic and F. Sanfilippo, Lattice $Q C D$ study of the radiative decays $J / \psi \rightarrow \eta_{c} \gamma$ and $h_{c} \rightarrow \eta_{c} \gamma$, JHEP 01 (2013) 28.

[14] C. Bacci, R. Baldini-Celio, et al., Multi-Hadronic Decays and Partial Widths of the J/psi (3100) Resonance Produced in $e^{+} e^{-}$Annihilation at ADONE, Phys.Lett.B 58 (1975) 471-474.

[15] B. Bartoli et al., Measurement of the J/psi (3100) Decay Widths Into $e^{+} e^{-}$and $m u^{+} m u^{-}$at ADONE, Lett.Nuovo Cim. 14 (1975) 73-81.

[16] R. Brandelik et al., DASP Collaboration, Results from DASP on $e^{+} e^{-}$annihilation between $3.1 \mathrm{GeV}$ and $5.2 \mathrm{GeV}$, Z.Phys.C 1 (1979) 233-256.

[17] Yu.S. Kalashnikova, A.V. Nefediev, X(3872) in the molecular model, Phys. Usp. 62 (2019) $6,568-595$

[18] V. V. Anashin et al., KEDR collaboration, Measurement of $D^{0}$ and $D^{+}$meson masses with the KEDR Detector, Phys.Lett.B 686 (2010) 84-90.

[19] K.Yu. Todyshev, Measuring the inclusive cross section of $e^{+} e^{-}$annihilation into hadrons in the preasymptotic energy range, Phys.Usp. 63 (2020) 9, 929-939.

[20] V.V. Anashin, et al., KEDR collaboration, Measurement of R between 1.84 and $3.05 \mathrm{GeV}$ at the KEDR detector, Phys.Lett. B 770 (2017) 174.

[21] V.V. Anashin, et al., KEDR collaboration, Precise measurement of Ruds and R between 1.84 and $3.72 \mathrm{GeV}$ at the KEDR detector, Phys.Lett. B 788 (2019) 42.

[22] J. Z. Bai et al., BES Collaboration, Measurements of the Cross Section for $e^{+} e^{-} \rightarrow$ Hadrons at Center-of-Mass Energies from 2 to $5 \mathrm{GeV}$, Phys. Rev. Lett. 88, 101802.

[23] D. Besson et al., CLEO Collaboration, Measurement of the total hadronic cross section in $e^{+} e^{-}$annihilation below $10.56 \mathrm{GeV}$, Phys. Rev. D 76, 072008. 